NTZ $97 / 31$

TPR-97-22

\title{
On the leading logarithmic evolution of the off-forward distributions
}

\author{
A.V. Belitsky ${ }^{1,2}$, B. Geyer ${ }^{3}$, D. Müller ${ }^{3}$, A. Schäfer ${ }^{2}$ \\ ${ }^{1}$ Bogoliubov Laboratory of Theoretical Physics, Joint Institute for Nuclear Research \\ 141980, Dubna, Russia \\ ${ }^{2}$ Institut für Theoretische Physik, Universität Regensburg \\ D-93040 Regensburg, Germany \\ ${ }^{3}$ Institute for Theoretical Physics, Center of Theoretical Science, Leipzig University \\ 04109 Leipzig, Germany
}

\begin{abstract}
We have found the analytical solution of the LO-evolution equation for off-forward distributions which arise in the processes of deeply virtual Compton scattering or exclusive production of mesons. We present the predictions for their evolution with an input distribution taken from recent bag model calculations.
\end{abstract}


1. Introduction. Recently, X. Ji proposed to explore deeply virtual Compton scattering (DVCS) (see Fig. [) to get deeper insight into the spin structure of the polarized nucleon and, namely, to determine the total quark angular momentum (spin and orbital angular momentum) in the nucleon [1, 2]. It turned out that this process is very interesting in its own right, since it allows to obtain information about some non-perturbative off-forward parton distributions (OFPDs), which are inaccessible in ordinary inclusive measurements such as deep inelastic scattering (DIS). Planning of experiments to measure DVCS is under way, but it is hampered by the lack of reliable theoretical predictions in the interesting $\left(\omega, \eta, Q^{2}\right)$-range. A crucial step towards this goal is to understand the $Q^{2}$-evolution of OFPD. In the present short note we, therefore, present the $Q^{2}$ evolution of the nonpolarized quark distribution function in the leading order (LO) approximation starting form MIT bag model predictions at a low energy scale [3].

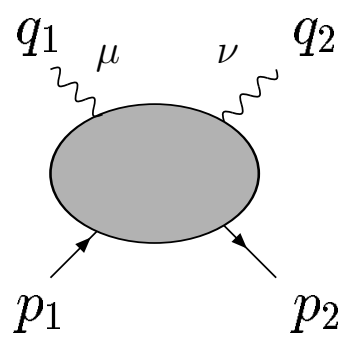

Figure 1: Generic form of the DVCS amplitude.

To make this paper self-contained, as much as possible, let us first recall the main points of the perturbative (LO) analysis [4, 1, 5, 6, 7, 8, 9, 10] of DVCS amplitude. In the configuration space such a Compton process, given by the following expression

$$
T^{\mu \nu}\left(\omega, \eta, Q^{2}\right)=i \int d^{4} z e^{i z Q}\left\langle p_{2}\left|T\left\{J^{\mu}(-z / 2) J^{\nu}(z / 2)\right\}\right| p_{1}\right\rangle,
$$

is dominated by singularities of the $T$-product of electromagnetic currents $J_{\mu}$ on the light-cone. In momentum space this dominance corresponds to the generalized Bjorken limit:

$$
-Q^{2} \rightarrow \infty, \quad P Q \rightarrow \infty, \quad \omega, \Delta^{2} \text {-fixed, }
$$

where the variables are defined as follows $Q=\frac{1}{2}\left(q_{1}+q_{2}\right), P=\left(p_{1}+p_{2}\right), \Delta=p_{1}-p_{2}=q_{2}-q_{1}$. DVCS is characterized by two scaling variables $\omega=-\frac{P Q}{Q^{2}}$ and $\eta=\frac{\Delta Q}{P Q}$ 四, the former is analogous to the Bjorken one in ordinary DIS while the latter gives the magnitude of the skewedness of the process in question and parametrizes different high-energy two-photon reactions according to the Table [1.

Notice that in general the unpolarized tensor $T^{\mu \nu}$ can be decomposed into four (three in the case of DVCS) independent kinematical structures that separately respect current conservation (similarly for polarized hadrons) [11. However, only two of them survive the general Bjorken limit, while the remaining ones are kinematically suppressed and contain higher twist contributions. In LO only one kinematical structure is independent, so that the longitudinal helicity amplitudes vanish. This is analogous to the Callan-Gross relation in unpolarized DIS. Motivated by the result that only transverse helicity amplitudes contribute in LO, we can write, e.g. for unpolarized scattering

$$
T\left(\lambda^{\prime}, \lambda\right)=\epsilon_{2}^{\mu}\left(\lambda^{\prime}\right) T_{\mu \nu} \epsilon_{1}^{\nu}(\lambda) \approx \frac{1}{2} \epsilon_{2}^{\nu}\left(\lambda^{\prime}\right) \epsilon_{1}^{\nu}(\lambda) \delta_{\lambda 1 / 2} \delta_{\lambda^{\prime} 1 / 2} T_{\mu \mu} .
$$




\begin{tabular}{|l|l|l|}
\hline DIS & $\Delta=0$ & $\eta=0$ \\
DVCS & $q_{2}^{2}=0$ & $\eta=1 / \omega$ \\
$\gamma^{*} N \rightarrow N l^{+} l^{-}$ & $q_{2}^{2}>0$ & $\eta \approx \cos \phi_{\mathrm{Br}}=\mathbf{p}_{1} \mathbf{Q} /\left|\mathbf{p}_{1}\right||\mathbf{Q}|$, Breit-frame \\
$\gamma^{*} \gamma^{*} \rightarrow H H$ & & $\eta \approx \cos \phi_{\mathrm{cm}}=\mathbf{p}_{1} \mathbf{Q} /\left|\mathbf{p}_{1}\right||\mathbf{Q}|$, center-of-mass frame \\
$\gamma^{*} \gamma^{*} \rightarrow M$ & $p_{1}=0$ & $\eta=1$ \\
\hline
\end{tabular}

Table 1: The value of the scaling variable $\eta$ for different two-photon processes.

Adding the surviving spin-dependent structure, we get円:

$$
\begin{aligned}
T_{\mu \mu}^{L O} & =\int_{-1}^{1} d t \sum_{i} Q_{i}^{2} q^{i}\left(t, \eta, \Delta^{2}\right)\left\{\frac{\omega}{\omega t-1+i 0}+\frac{\omega}{\omega t+1-i 0}\right\} \\
T_{[\mu \nu]}^{L O} & =\frac{i \epsilon_{\mu \nu P Q}}{2 P Q} \int_{-1}^{1} d t \sum_{i} Q_{i}^{2} \Delta q^{i}\left(t, \eta, \Delta^{2}\right)\left\{\frac{\omega}{\omega t-1+i 0}-\frac{\omega}{\omega t+1-i 0}\right\} .
\end{aligned}
$$

Here the off-forward distributions are defined in terms of the light-cone Fourier transformation of the renormalized twist-2 light-ray operators:

$$
\left\{\begin{array}{c}
q \\
\Delta q
\end{array}\right\}\left(t, \eta, \Delta^{2}, \mu^{2}\right)=\int \frac{d \kappa}{2 \pi} e^{\frac{i}{2} \kappa t P_{+}}\left\langle p_{2}\left|\bar{\psi}(-\kappa / 2 n)\left\{\begin{array}{c}
\gamma_{+} \\
\gamma_{+} \gamma_{5}
\end{array}\right\} \psi(\kappa / 2 n)\right|_{\mu^{2}} \mid p_{1}\right\rangle_{\Delta_{+}=\eta P_{+}}
$$

and we have omitted the path ordered link factor which ensures gauge invariance. The integration range of the variable $t$ in Eq. (2) results from the spectral properties of the off-forward distribution amplitude that can be derived in a straightforward manner with the help of Jaffe's approach [13] for studying the support properties of parton densities. In our conventions the variable $t$ has no direct interpretation as longitudinal momentum fraction, but it has the advantage that its region is process independent: $-1 \leq t \leq 1$. The same feature is appropriate to the quark $\mathcal{F}_{\zeta}^{a}(x)(0 \leq x \leq 1)$ and anti-quark $\mathcal{F}_{\zeta}^{\bar{a}}(x)(-1 \leq x \leq 0)$ non-forward distributions introduced by Radyushkin [5]. The $\zeta$-dependent limits appear only when one attempts to combine them into one function $\mathcal{F}_{\zeta}^{a}(x)=\theta(x) \theta(\bar{x}) \mathcal{F}_{\zeta}^{a}(x)-\theta(\zeta-x) \theta(x+1-\zeta) \mathcal{F}_{\zeta}^{\bar{a}}(\zeta-x)$ which is exactly the OFPD introduced above in Eq. (4) in different notations. The non-diagonal distribution ${ }^{2} f^{g}\left(x_{1}, x_{2}\right)$ of Collins, Frankfurt and Strikman [6] is parametrized in terms of the momentum fraction of the considered partons and consequently their limits depend on the skewedness of the processes. Thus, the momentum fractions of the incoming and outgoing partons are $x=\frac{t+\eta}{1+\eta}$, and $x-\zeta=\frac{t-\eta}{1+\eta}$, respectively, with $\zeta=\frac{2 \eta}{1+\eta}$. For $t>\eta$ both momentum fractions are positive and, therefore, a quark field with longitudinal momentum $x p_{1}$ arise from the incoming hadron and enters with momentum $(x-\zeta) p_{1}$ into the outgoing hadron. For $-\eta>t$ both momentum fractions are negative and the analogous picture for two anti-quarks holds true. In these regions, the corresponding offforward quark and anti-quark distributions merge in the forward case to the well-known quark and anti-quark distributions with support $0 \leq t \leq 1$ and $-1 \leq t \leq 0$, respectively. For $-\eta<t<\eta$, $x$ is positive while $x-\zeta$ is negative, so that both partons come from the same hadron. Here the OFPD looks like a distribution amplitude. Indeed, inserting the vacuum and one-meson state

\footnotetext{
${ }^{1}$ Recently, all NLO coefficient functions [12, 10] as well as $\alpha_{s}$-corrections to the eigenfunctions of the two-loop $Q Q$ - and $Q G$-evolution kernels became available.

${ }^{2}$ In the Radyushkin's conventions $f^{g}(x, x-\zeta)=\widetilde{\mathcal{F}}_{\zeta}^{g}(x) / x(x-\zeta)[5]$.
} 
in the definition (47) instead of incoming and outgoing nucleon state, we recover the definition of the meson distribution amplitude depending on the momentum fraction $0 \leq x=(1+t) / 2 \leq 1$, where $\eta=1$ [14]. Thus, OFPDs are hybrids and their probability interpretation depends upon the interplay between the respective values for $t$ and $\eta$.

Finally, to end up with this introduction, we point out a few difficulties of the measurement of the OFPDs in DVCS. DVCS can be explored in the reaction

$$
e^{ \pm} N \rightarrow e^{ \pm} \gamma N
$$

However, DVCS and Bethe-Heitler (BH) sub-processes have the same final states. Hence, they interfere and the measured cross section contains the squares of pure DVCS and BH parts as well as the interference term:

$$
d \sigma \propto\left|T_{\mathrm{DVCS}}\right|^{2}+\left|T_{\mathrm{BH}}\right|^{2}+\left(T_{\mathrm{DVCS}}^{*} T_{\mathrm{BH}}+T_{\mathrm{BH}}^{*} T_{\mathrm{DVCS}}\right) .
$$

Fortunately, the contribution from the $\mathrm{BH}$ process is known from the measurements of the nucleon form factors. In unpolarized or double spin experiments DVCS amplitude is given as convolution [compare with Eqs. (2) and (3)], so that a direct extraction of the OFPDs seems to be very

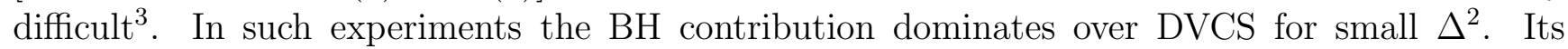
importance decrease, however, with increasing $\Delta^{2}$ 囵. In single spin experiments a non-vanishing cross section arises from the imaginary part of the amplitude. Since BH has only a real part, the single spin asymmetry is proportional to the imaginary part of the DVCS amplitude, so that the OFPDs can be measured directly at the point $t= \pm 1 / \omega$. Hopefully, as a first step in the direction of DVCS measurements, the predicted scaling behaviour in the generalized Bjorken regime will be investigated at CEBAF [1], 3].

2. Evolution equation. The evolution of OFPDs arises technically from the renormalization procedure for the light-ray operators [15], which satisfy in the non-singlet channel the well-known renormalization group equation [16, 17, 18, 19]. Employing the definition (4), it is straightforward to derive from the latter the evolution equation for the OFPD:

$$
Q^{2} \frac{d}{d Q^{2}} q^{\mathrm{NS}}\left(t, \eta, \Delta^{2}, Q^{2}\right)=\frac{\alpha_{s}}{2 \pi} \int_{-1}^{1} \frac{d t^{\prime}}{|\eta|}\left[V\left(\frac{t}{\eta}, \frac{t^{\prime}}{\eta}\right)\right]_{+} q^{\mathrm{NS}}\left(t^{\prime}, \eta, \Delta^{2}, Q^{2}\right) .
$$

In the restricted region $\left|t, t^{\prime}\right| \leq 1$ the kernel $V\left(t, t^{\prime}\right)$ coincides with the Efremov-RadyushkinBrodsky-Lepage (ER-BL) one [14]:

$$
V_{\mathrm{ER}-\mathrm{BL}}(x, y)=\left.V(2 x-1,2 y-1)\right|_{0 \leq x, y \leq 1} .
$$

Moreover, the continuation to the whole $t, t^{\prime}$-plane is unique [⿶], so that the extended evolution kernel $V\left(t, t^{\prime}\right)$ can be easily recovered from the corresponding ER-BL analogues. From the support restrictions and charge conjugation symmetry a representation was derived in [20], which is valid in leading order:

$$
V\left(t, t^{\prime}\right)=\Theta_{11}^{0}\left(t-t^{\prime}, t-1\right) \mathcal{V}\left(t, t^{\prime}\right)+\left(t \rightarrow-t, t^{\prime} \rightarrow-t^{\prime}\right),
$$

where $\Theta_{11}^{0}\left(x_{1}, x_{2}\right)=\left(\theta\left(x_{1}\right) \theta\left(-x_{2}\right)-\theta\left(x_{2}\right) \theta\left(-x_{1}\right)\right) /\left(x_{1}-x_{2}\right)$, and $\mathcal{V}\left(t, t^{\prime}\right)$ is an analytic function of $t$ and $t^{\prime}$. Thus, the extended kernel can be obtained from the exclusive ER-BL one by replacing

\footnotetext{
${ }^{3}$ The imaginary part of the LO scattering amplitude (3) is expressed in terms of the OFPDs at $t= \pm 1 / \omega$, while the real part is given as a Hilbert transformation and can be inverted in principle.
} 
the ordinary $\theta$-functions in front of $\mathcal{V}\left(t, t^{\prime}\right)$ by the generalized ones, namely, $\theta\left(t-t^{\prime}\right) /\left(1-t^{\prime}\right) \rightarrow$ $\Theta_{11}^{0}\left(t-t^{\prime}, t-1\right)$. For concrete examples the reader is referred to Ref. [10]. Beyond LO, further contributions appear in Eq. (9), but they are not of relevance for our present consideration.

For $\eta \rightarrow 0$ the evolution equation (7) turns into the DGLAP one with a splitting function given by the limit:

$$
P(z)=\lim _{\eta \rightarrow 0} \frac{1}{|\eta|}\left[V\left(\frac{z}{\eta}, \frac{1}{\eta}\right)\right]_{+} .
$$

3. Solution of the evolution equation. The evolution equation analogous to Eq. (7), but for gluons, was attempted to be integrated numerically [8]. However, it remains an important issue to find an analytical solution. The way to do that is based on the use of the conformal invariance of the free field theory which ensures that conformal 2-particle operators do not mix under renormalization at LO [14, 21, 22, 23, 10]. Such conformal operators are given in terms of Gegenbauer polynomials $Q_{\mathcal{O}_{j l}}=\bar{\psi}\left(i \partial_{+}\right)^{l} \gamma_{+} C_{j}^{\frac{3}{2}}\left(\stackrel{\leftrightarrow}{D}+/ \partial_{+}\right) \psi$ and their off-forward matrix elements can be obtained from the moments

$$
q_{j}\left(\eta, \Delta^{2}, Q^{2}\right)=\eta^{j} \int_{-1}^{1} d t C_{j}^{\frac{3}{2}}\left(\frac{t}{\eta}\right) q\left(t, \eta, \Delta^{2}, Q^{2}\right)=\frac{2}{P_{+}^{j+1}}\left\langle\left.\left. p_{2}\right|^{Q} \mathcal{O}_{j j}(0)\right|_{\mu^{2}=Q^{2}} \mid p_{1}\right\rangle .
$$

In the following, we restrict ourselves to the flavour non-singlet channel since up to now no estimation of the off-forward gluon distribution has been performed yet as long as available strong interaction models do not contain the gluon fields at all. The generalization of our results to the singlet channel is straightforward. The only complication which is due to quark-gluon mixing can be treated in the same way as in DIS. For $\eta=1$ it is well-known that the Gegenbauer polynomials diagonalize the ER-BL-kernel. Moreover, the support of the extended evolution kernel ensures this property for arbitrary $\eta$ :

$$
\int_{-1}^{1} d t C_{j}^{\frac{3}{2}}\left(\frac{t}{\eta}\right)\left[V\left(\frac{t}{\eta}, \frac{t^{\prime}}{\eta}\right)\right]_{+}=\gamma_{j} C_{j}^{\frac{3}{2}}\left(\frac{t^{\prime}}{\eta}\right)
$$

The remaining problem is to find the inverse Mellin transformation of the moments (11). The support property' of $q^{\mathrm{NS}}\left(t, \eta, Q^{2}\right)$ allows to expand it with respect to an appropriate complete set of polynomials $C_{k}^{\nu}(t)$ which are orthogonal in the domain $-1 \leq t \leq 1$ with the weight function $w(t \mid \nu)=\left(1-t^{2}\right)^{\nu-\frac{1}{2}}$ :

$$
q^{\mathrm{NS}}\left(t, \eta, Q^{2}\right)=\sum_{j=0}^{\infty} \frac{w\left(t \mid \frac{3}{2}\right)}{N_{j}\left(\frac{3}{2}\right)} C_{j}^{\frac{3}{2}}(t) a_{j}\left(\eta, Q^{2} \mid \frac{3}{2}\right)
$$

where $N_{j}(\nu)=2^{-2 \nu+1} \frac{\Gamma^{2}\left(\frac{1}{2}\right) \Gamma(2 \nu+j)}{\Gamma^{2}(\nu)(\nu+j) j !}$ is a normalization factor. It is straightforward to calculate the expansion coefficients in terms of the conformal moments (11):

$$
a_{j}\left(\eta, Q^{2} \mid \frac{3}{2}\right)=\sum_{k=0}^{j} a_{j k}\left(\eta \mid \frac{3}{2}\right) q_{k}^{\mathrm{NS}}\left(\eta, Q_{0}^{2}\right) \exp \left\{\gamma_{k} \int_{Q_{0}^{2}}^{Q^{2}} \frac{d \sigma}{\sigma} \frac{\alpha_{s}(\sigma)}{2 \pi}\right\},
$$

\footnotetext{
${ }^{4}$ In the subsequent discussion we omit, for brevity, the dependence of the distributions on the momentum transferred squared.
} 
where $\gamma_{j}=C_{F}[-2 \psi(j+2)+2 \psi(1)+1 /(j+1)-1 /(j+2)+3 / 2]$ are the well-known forward non-singlet anomalous dimensions, and $a_{j k}\left(\eta \mid \frac{3}{2}\right)$ can be written in a very compact manner by employing the definition of hypergeometric functions:

$$
\begin{aligned}
a_{j k}(\eta \mid \nu) & =\int_{-1}^{1} d t \frac{w(t \mid \nu)}{\eta^{k} N_{k}(\nu)} C_{k}^{\nu}(t) C_{j}^{\nu}(\eta t) \\
& =\frac{1}{2} \theta_{j k}\left[1+(-1)^{j-k}\right] \frac{(-1)^{\frac{j-k}{2}} \Gamma\left(\nu+\frac{j+k}{2}\right)}{\Gamma(\nu+k) \Gamma\left(1+\frac{j-k}{2}\right)}{ }_{2} F_{1}\left(\begin{array}{c}
\frac{k-j}{2}, \nu+\frac{j+k}{2} \\
\nu+k+1
\end{array} \mid \eta^{2}\right) .
\end{aligned}
$$

Let us turn to the consideration of the limiting cases. Since ${ }_{2} F_{1}(-n, a+n ; a+1 ; 1)=\delta_{n 0}$, we obtain for $\eta=1$ :

$$
a_{k}\left(1, Q^{2} \mid \frac{3}{2}\right)=q_{k}^{\mathrm{NS}}\left(1, Q_{0}^{2}\right) \exp \left\{\gamma_{k} \int_{Q_{0}^{2}}^{Q^{2}} \frac{d \sigma}{\sigma} \frac{\alpha_{s}(\sigma)}{2 \pi}\right\}
$$

and, therefore, from Eq. (13) we recover the well-known LO result for the evolution of the meson distribution amplitude [14]. In the limit $\eta \rightarrow 0$, the solution of the DGLAP equation expanded with respect to Gegenbauer polynomials $C_{k}^{\frac{3}{2}}$, has the following coefficients

$$
a_{j}\left(0, Q^{2} \mid \frac{3}{2}\right)=\sum_{\substack{k=0 \\ k-j \text { even }}}^{j} \frac{(-1)^{\frac{j-k}{2}} \Gamma\left(\frac{3+j+k}{2}\right)}{\Gamma\left(\frac{3+2 k}{2}\right) \Gamma\left(\frac{2+j-k}{2}\right)} q_{k}^{\mathrm{NS}}\left(0, Q_{0}^{2}\right) \exp \left\{\gamma_{k} \int_{Q_{0}^{2}}^{Q^{2}} \frac{d \sigma}{\sigma} \frac{\alpha_{s}(\sigma)}{4 \pi}\right\} .
$$

In this limit conformal moments coincide up to an overall normalization with the usual ones in DIS:

$$
q_{k}^{\mathrm{NS}}\left(0, Q_{0}^{2}\right)=\frac{2^{k} \Gamma(\nu+k)}{k ! \Gamma(\nu)} \int_{0}^{1} d x x^{k}\left[q^{\mathrm{NS}}\left(x, 0, Q_{0}^{2}\right)+(-1)^{k} q^{\mathrm{NS}}\left(-x, 0, Q_{0}^{2}\right)\right]
$$

For asymptotically large $Q^{2}$ all conformal moments with $k>0$ will be suppressed due to nonzero anomalous dimensions and, consequently, only $j$-even expansion coefficients survive in this limit:

$$
\begin{aligned}
a_{j}^{\text {as }}\left(\eta \mid \frac{3}{2}\right) & =\lim _{Q \rightarrow \infty} a_{j}\left(\eta, Q \mid \frac{3}{2}\right) \\
& =\frac{(-1)^{\frac{j}{2}} \Gamma\left(\frac{3+j}{2}\right)}{\Gamma\left(\frac{3}{2}\right) \Gamma\left(\frac{2+j}{2}\right)}{ }_{2} F_{1}\left(\begin{array}{c}
-j / 2,(3+j) / 2 \\
5 / 2
\end{array} \mid \eta^{2}\right) q_{0}\left(\eta, Q_{0}^{2}\right) \quad \text { for } j \text { even. }
\end{aligned}
$$

It is not hard to check that they belong to the following asymptotic off-forward distribution which was originally found in Ref. [5]:

$$
q^{\mathrm{as}}(t, \eta) \propto \frac{1}{|\eta|} \theta\left(1-\frac{t^{2}}{\eta^{2}}\right)\left(1-\frac{t^{2}}{\eta^{2}}\right) .
$$

It turns into $\delta(t)$ when $\eta \rightarrow 0$. From the asymptotic form found so far, we can argue that, in general, OFPDs will be enhanced in the region $0 \leq|t|<\eta$ and suppressed for $\eta<|t| \leq 1$ when evolved upwards in $Q^{2}$. To demonstrate these effects in a clear manner, we choose a $\eta$-independent toy-model input distribution $3 / 4\left(1-t^{2}\right.$ ) (which is in fact unphysical for arbitrary $\eta$ ). For $\eta=1$ this 


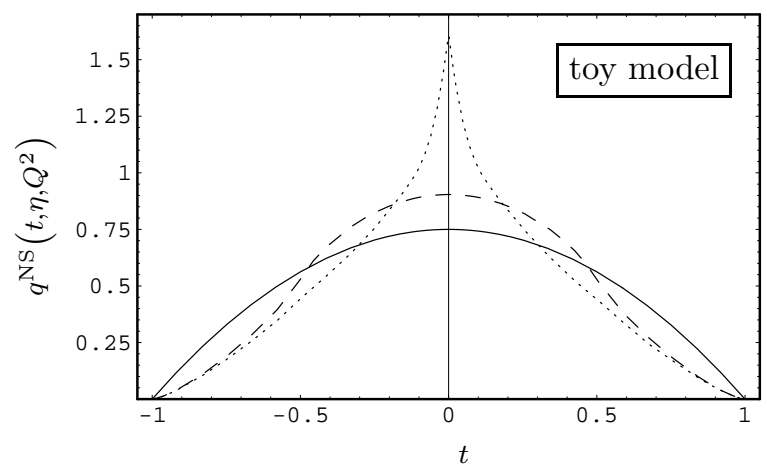

Figure 2: The toy input OFPD (solid line) given at $Q^{2}=0.5 \mathrm{GeV}^{2}$ is evolved up to $Q^{2}=5 \mathrm{GeV}^{2}$, where $\Lambda_{\mathrm{QCD}}=220 \mathrm{MeV}$. The dashed and dotted lines show the evolution for $\eta=0.5$ and $\eta=0$, respectively, while for $\eta=1$ the input distribution does not evolve since it has already the asymptotic shape.

distribution possesses already the asymptotic form, while for smaller $\eta$ 's the evolution provides the mentioned behaviour as illustrated in Fig. 2. The evolution has been done with the help of Eqs. (13)-(15), while the conformal moments of our toy distribution at the input scale $Q^{2}=0.5 \mathrm{GeV}^{2}$ was computed from Eq. (11). Taking the first 80 terms in Eq. (13) the OFPD was evolved to $Q^{2}=5 \mathrm{GeV}^{2}$ for $\eta=\{5 / 10,0\}$ and $t=0, \pm 1 / 20, \pm 2 / 20, \ldots, 1$. To avoid numerical problems arising from the oscillation of the Gegenbauer polynomials the calculation were done exactly; and, finally, the result was interpolated to a smooth function.

4. $Q^{2}$-evolution of the bag model motivated OFPD. A first non-perturbative estimation of the off-forward valence quark distribution function has been done using the MIT bag model[ [3]. In this model calculation it turned out that at a scale $\mu_{\mathrm{bag}}^{2} \simeq 0.2 \mathrm{GeV}^{2}$ the $\eta$-dependence of the off-forward $u$ and $d$ quark distributions is extremely weakf and these functions vanish for negative $t$ as well as for $t \rightarrow 1$. (The nonzero result of the calculated $t$-dependence in the vicinity of unity is a model artifact, which reflects the fact that the incoming and outgoing protons are not good momentum eigenstates.) For positive $t$ the distributions are positive with a maximum at $t \approx 0.4$. To be able to satisfy the sum rules which are obeyed by the off-forward distributions, namely, the first two moments should be independent of the skewedness parameter $\eta$

$$
\int_{-1}^{1} d t\left\{\begin{array}{l}
1 \\
t
\end{array}\right\} q\left(t, \eta, Q^{2}\right)=\frac{2}{P_{+}^{2}}\left\langle p_{2}\left|\left\{\begin{array}{c}
P_{+} J^{+}(0) \\
Q_{\Theta}^{++}(0)
\end{array}\right\}\right| p_{1}\right\rangle,
$$

$\left({ }^{Q} \Theta^{\mu \nu}\right.$ is a quark part of the energy-momentum tensor), we neglect the $\eta$-dependence altogether, which is not far from the bag model results. Since the main goal of the present study is to acquire some intuition about the evolution properties of OFPD, we do not pursue the aim for construction of more realistic models; rather motivated by the given results, we take a very simple parametrization of the OFPD in the non-singlet channel, namely

$$
q^{\mathrm{NS}}\left(t, \eta, Q_{0}^{2}=\mu_{\text {bag }}^{2}\right)=60 \theta(t) t^{2}(1-t)^{3},
$$

\footnotetext{
${ }^{5}$ To be general we have not specified the index $\nu$ : for the quark operators $\nu=3 / 2$, for gluons $\nu=5 / 2$.

${ }^{6}$ Quite recently there appeared a paper 24 with evaluation of the OFPDs in the instanton inspired chiral quark-soliton model of the nucleon. However, it is not possible to use their results here as only flavour singlet quark density was considered. Moreover, flavour non-singlet combination is $1 / N_{c}$-suppressed in this approach.

${ }^{7}$ This is in contrast with the result of Ref. [24], where the $\eta$-dependence is rather strong.
} 
with a first moment normalized to unity.

In Fig. 3, we evolve this input as described previously (we took also discrete values for $\eta=$ $0,1 / 10, \ldots, 1$ and interpolated the result with respect to $t$ and $\eta$ ) up to the scales $Q^{2}=2 \mathrm{GeV}^{2}$, $Q^{2}=200 \mathrm{GeV}^{2}$, and asymptotically large $Q^{2}$; for other parameters, we set $N_{f}=3, \Lambda_{\mathrm{QCD}}=$ $220 \mathrm{MeV}$ in $\alpha_{s}\left(Q^{2}\right)$. As expected from our previous discussion, the distribution spreads in $t$ for larger value of $\eta$ and shrinks for smaller ones. These graphs clearly show that during the
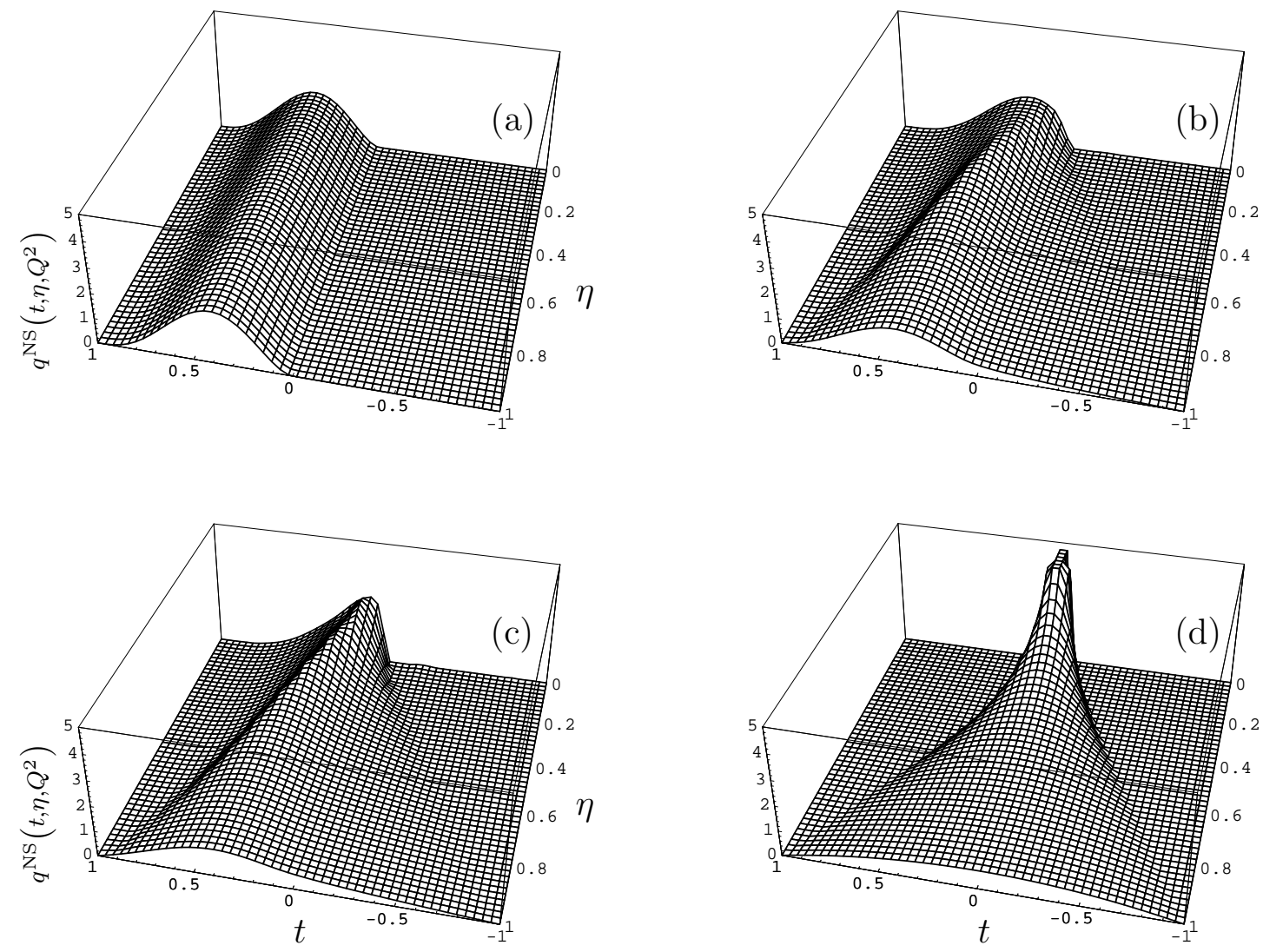

Figure 3: The evolution of the bag model motivated OFPD (22) for $\Lambda_{\mathrm{QCD}}=220 \mathrm{MeV}$, where the scales are $Q_{0}^{2}=\mu_{\text {bag }}^{2}$ (a), $Q^{2}=2 \mathrm{GeV}^{2}$ (b), $Q^{2}=200 \mathrm{GeV}^{2}$ (c), and asymptotically large $Q^{2}$ (d).

evolution the large- $\eta$ part spreads over the whole range of the momentum fraction while the small- $\eta$ distribution is pushed towards $t \rightarrow 0$ and concentrates in the vicinity of zero. Since we have omitted in the present analysis the gluon sector, we can expect that taking it into account we will get, probably, an even more enhanced function in the small- $t$ (and $\eta \rightarrow 0$ ) region for moderate values of $Q^{2}$. As is easily seen from the above figures with growing scale the OFPD approaches the asymptotic form. Namely, for $\eta \rightarrow 1$ it takes the $\left(1-t^{2}\right)$-shape of the asymptotic distribution amplitude, while for small $\eta$ it becomes $\delta(t)$. For intermediate $\eta$ it smoothly interpolates between these limits. This is a quite general feature that should be obeyed by any reasonable (read physical) off-forward parton density.

We would like to thank A.V. Radyushkin for careful reading of the manuscript and useful comments and O.V. Teryaev for discussions. A.B. was supported by the Russian Foundation 
for Fundamental Research, grant N 96-02-17631 and Deutsche Physikalische Gesellschaft Bayern. D.M. was financially supported by the Deutsche Forschungsgemeinschaft (DFG).

\section{References}

[1] X. Ji, Phys. Rev. Lett. 78 (1997) 610; Phys. Rev. D 55 (1997) 7114.

[2] X. Ji, Lorentz symmetry and the internal structure of the nucleon, hep-ph/9710290.

[3] X. Ji, W. Melnitchouk, X. Song, A study of off-forward parton distributions, hep-ph/9702379.

[4] D. Müller, D. Robaschik, B. Geyer, F.-M. Dittes, J. Hořejši, Fortschr. Phys. 42 (1994) 101.

[5] A.V. Radyushkin, Phys. Lett. B 380 (1996) 417; Phys. Lett. B 385 (1996) 333; Phys. Rev. D 56 (1997) (in print), hep-ph/9704207;

I.I. Balitsky, A.V. Radyushkin, Light-ray evolution equations and leading-twist parton helicitydependent non-forward distributions, hep-ph/9706410.

[6] J.C. Collins, L.L. Frankfurt, M. Strikman, Phys. Rev. D 56 (1997) 2982.

[7] J. Blümlein, B. Geyer, D. Robaschik, Phys. Lett. B 406 (1997) 161.

[8] L. Frankfurt, A. Freund, V. Guzey, M. Strikman, Nondiagonal parton distribution in the leading logarithmic approximation, hep-ph/9703449.

[9] Z. Chen, Non-forward and unequal mass virtual compton scattering, hep-ph/9705279.

[10] A.V. Belitsky, D. Müller, Predictions from conformal algebra for the deeply virtual Compton scattering, hep-ph/9709379.

[11] W. Lu, Form factor description of the non-collinear Compton scattering tensor, hepph/9707310.

[12] X. Ji, J. Osborne, One-loop QCD corrections to the deeply-virtual Compton scattering: the parton helicity-independent case, hep-ph/9707254.

[13] R.L. Jaffe, Nucl. Phys. B 229 (1983) 205.

[14] S.J. Brodsky, G.P. Lepage, Phys. Lett. B 87 (1979) 359; Phys. Rev. D 22 (1980) 2157;

A.V. Efremov, A.V. Radyushkin, JINR preprint E2-11983, Dubna (October 1978); Theor. Math. Phys. 42 (1980) 97; Phys. Lett. B 94 (1980) 245.

[15] S.A. Anikin, O.I. Zavialov, Ann. Phys. 116 (1978) 135.

[16] B. Geyer, Czech. J. Phys. B 32 (1982) 645.

[17] I.I. Balitsky, Phys. Lett. B 124 (1983) 230.

[18] T. Braunschweig, B. Geyer, D. Robaschik, Ann. Phys. (Leipzig) 44 (1987) 403.

[19] I.I. Balitsky, V.M. Braun, Nucl. Phys. B 311 (1989) 541. 
[20] F.-M. Dittes, D. Müller, D. Robaschik, B. Geyer, Phys. Lett. B 209 (1988) 325.

[21] Yu.M. Makeenko, Sov. J. Nucl. Phys. 33 (1981) 440.

[22] Th. Ohrndorf, Nucl. Phys. B 198 (1982) 26.

[23] D. Müller, Phys. Rev. D 49 (1994) 2525.

[24] V.Yu. Petrov, P.V. Pobylitsa, M.V. Polyakov, I. Börnig, K. Goeke, C. Weiss, Off-forward quark disrtibutions of the nucleon in the large $N_{c}$ limit, hep-ph/9710270. 